\title{
The improved method in agarose gel electrophoresis of nucleic acid
}

\author{
Tingting Yang, Qi Tang* \\ Key Laboratory of Antibody Technique of National Health and Family Planning Commission, Nanjing Medical University, \\ Nanjing, Jiangsu 211166, China
}

\begin{abstract}
In most molecular experiments, nucleic acids are subjected to agarose gel electrophoresis to determine the size of the molecule. The addition of a nucleic acid dye allows the nucleic acid to be detected under the UV image system after running the gel, so the nucleic acid dye is an integral part of the electrophoresis experiment. But when considering the mutagenicity and toxicity of nucleic acid dyes, one must be careful to insure the proper disposal of experimental waste. In this article, a new usage of nucleic acid dye in agarose gel electrophoresis is described where the nucleic acid dyes were added to the loading buffer and nucleic acid marker buffer. The results show that this method has advantages as: a smaller amount of dye can be used, there is less time in contact with the dye, and its operation is easier and reduces toxicity damage. Also the bands showed a much clearer image, having a lower background value. The improved method shows better results with lower toxicity and is superior to the traditional method.
\end{abstract}

Keywords: agarose gel electrophoresis, nucleic acid dyes

\section{INTRODUCTION}

Agarose gel electrophoresis is a common method for detecting the size of a nucleic acid sequences and judging whether they are smaller or larger than expected, whether there are other miscellaneous sequences, and whether the polymerase chain reaction (PCR) results are satisfactory ${ }^{[1]}$. As the speeds of migration are different when nucleic acid sequences with different size are performed electrophoresis at the same voltage, the nucleic acid sequences are separated accordingly. Nucleic acid dyes will then be added to the agarose gel for the color of the image ${ }^{[2]}$. Most nucleic acid dye, especially ethidium bromide (EB) has strong mutagenicity and toxicity, and EB is considered a hazardous waste and must be disposed of appropri-

*Correspondence to: Qi Tang, Key Laboratory of Antibody Technique of National Health and Family Planning Commission, Nanjing Medical University, Nanjing, Jiangsu 211166, China. TEL: 0086-25-86869411. E-mail: qitang@njmu.edu.cn.

The authors have declared no conflict of interests. ately ${ }^{[3]}$. Therefore one must be especially careful when handling agarose gels containing it. Although there are some alternatives to EB such as gel red, gel green, GoldViewna and the like ${ }^{[4,5]}$, their safety is still relative, so an effective method to maintain less contact with the nucleic acid dye and to get clearer imaging is required. This study showed a new reagent loading method, which reduced direct contact with the dye, cut down the dosage, and can be widely used for nucleic acid gel electrophoresis.

\section{MATERIAL AND METHODS}

\section{Reagents and apparatus}

Agarose (cat\#75510-019, Invitrogen, USA), $10,000 \times$ Solar Red nucleic acid dye (Solarbio, China), DNA loading buffer (cat\#9157, TaKaRa, Japan), DL500 DNA Marker (cat\#3590Q, TaKaRa, Japan) and DL5000 DNA Marker (cat\#3428Q, TaKaRa, Japan) are necessary solution. $1 \times \mathrm{TAE}$ running buffer including ${ }^{[6]}$ : Tris base $242 \mathrm{~g}$, EDTA $100 \mathrm{~mL}$ (concentration: $0.5 \mathrm{~mol} / \mathrm{L} ; \mathrm{pH} 8.0$ ), acetic acid $57.1 \mathrm{~mL}$, 
add deionized water to volume $50 \mathrm{~L}$. Nucleic acid electrophoresis apparatus (Bio-Red, USA) and nucleic acid electrophoresis tank (Tanon, China) used for running programs, ChemiDoc ${ }^{\mathrm{TM}} \mathrm{XRS}+\mathrm{UV}$ image system (Bio-Rad, USA) used for imaging.

\section{Nucleic acid sequence samples}

Two different length nucleic acid sequences of PCR were taken. Gastric cancer cells: AGS, MKNl, SUN719, MKN45 were cultured in DMEM supplemented with $10 \%$ FBS, and $1 \%$ penicillin-streptomycin solution at $37{ }^{\circ} \mathrm{C}, 5 \% \mathrm{CO}_{2}$ in a humidified atmosphere. The cells were collected when they grew to more than $80 \%$ confluency, then total RNAs were extracted from four different gastric cancer cells and were reverse transcribed into first strand cDNA with HiScript 1st Strand cDNA Synthesis Kit (cat\#R111-01/02, Vazyme, China). PCRs were performed by using Taq DNA Polymerase Kit (cat\#P101-01/02/03, Vazyme, China) with two different primers, and used the cDNAs from AGS, MKN1, SUN719, MKN45 cells as templates, respectively. The target products of the PCR were 260 base pairs (bp) (fragment 1) and 1,429 bp (fragment 2), and were used as the main detection sequences for electrophoresis.

\section{The improved method of agarose gel electrophoresis}

The traditional method is based on a protocol laid down by widely published articles ${ }^{[1,7]}$, whereby gel red is added to agarose gel for electrophoresis. The improved method is as follows.

(1) Put $40 \mathrm{~mL}$ of TAE working solution into a conical flask, add $0.8 \mathrm{~g}$ of agarose in it and mix them thoroughly, cover the opening with foil, melt the agarose by heating them in a microwave oven for a short time and for many times.

(2) When all the feather-liked agarose particles are dissolved, shake off the bubbles in the solution and pour into a $6 \mathrm{~cm} \times 10 \mathrm{~cm}$ mini-sub cell GT8 gel rig, and plug in a large hole gel comb to create the wells.

(3) Cool the agarose gel at room temperature for 30 min to solidify, remove the comb, and transfer the gel into the electrophoresis tank.

(4) Add the gel red separately to $10 \times$ loading buffer, DL500 DNA Marker and DL5000 DNA Marker, then $9 \mu \mathrm{L}$ nucleic acid fragment solution was mixed with $1 \mu \mathrm{L} 10 \times$ loading buffer containing gel red. Loading buffer was used to help track how far the DNA sample has traveled, and to help the sample sink into the gel.

(5) Add the samples to the wells of agarose gel by 10 or $8,4,2,1$ and $0.5 \mu \mathrm{L}$ per well, with DL500 DNA Marker and DL5000 DNA Marker solution at $5 \mu \mathrm{L}$ per well.
(6) Add TAE buffer to the electrophoresis tank until covering the surface of gel.

(7) Maintain $130 \mathrm{~V}$ at a constant voltage running for around $30 \mathrm{~min}$, so that the front edge of the bromophenol blue tracking dye has migrated to a point that is $70 \%$ down the gel.

(8) Expose the gel under the ChemiDoc ${ }^{\mathrm{TM}} \mathrm{XRS}+\mathrm{UV}$ image system.

\section{Statistical analysis}

The data's statistical analysis was performed using the SPSS 18.0 statistical software package (SPSS Inc, Chicago, IL, USA). The paired $t$ test was used to determine the significance of the optical density value between the two methods.

\section{RESULTS AND DISCUSSION}

The experiment was repeated five times, each time using the same nucleic acid sequence, $2 \%$ agarose gel concentration, and ran at $130 \mathrm{~V}$ continuing for $30 \mathrm{~min}$. Except for the gel red dye addition, the other conditions were consistent between the traditional method and the improved method. The results showed that fragment 1 was around $250-300 \mathrm{bp}$, the bands were clearer, and the background value was lower in the improved method (Fig.1A, 1B). The fragment 2 appeared at around 1,000-1,500 bp, with the bands much clearer in the improved method compared with the traditional method (Fig.1C, 1D). The paired samples $t$-test analyzing the optical density value of the bands showed that the $P$ value of two groups was < 0.05 (Fig.1E, 1F). The difference between the results of the two methods was statistically significant. When two fragments were added at $8,4,2,1,0.5 \mu \mathrm{L}$ volumes for both methods, the results showed that the stripes in the improved method were detected even in the smallest volume $0.5 \mu \mathrm{L}$ (Fig.1G, 1H). The image of stripes obtained through improved method was clearer and more distinct when compared with those by traditional method. Interestingly, according to the results, the images of the longer sequence were clearer than those of the short nucleic acid sequence in the improved method.

In this experiment, gel red was selected as the nucleic acid dye. This was due to widespread evidence showing, when employed in UV transillumination, gel red has the greatest sensitivity compared to other dyes and is less toxic than $\mathrm{EB}^{[8]}$. This experiment was based on the original nucleic acid agarose gel electrophoresis method, with modification of the usage of nucleic acid dye which is certain carcinogenic and mutagenic. In this study, the authors examined two lengths of nu- 
A

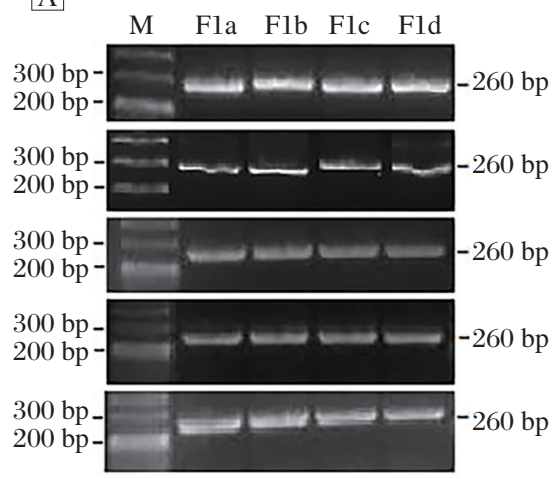

D

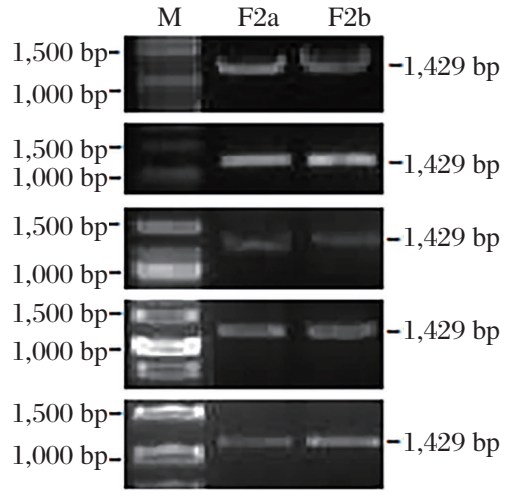

G
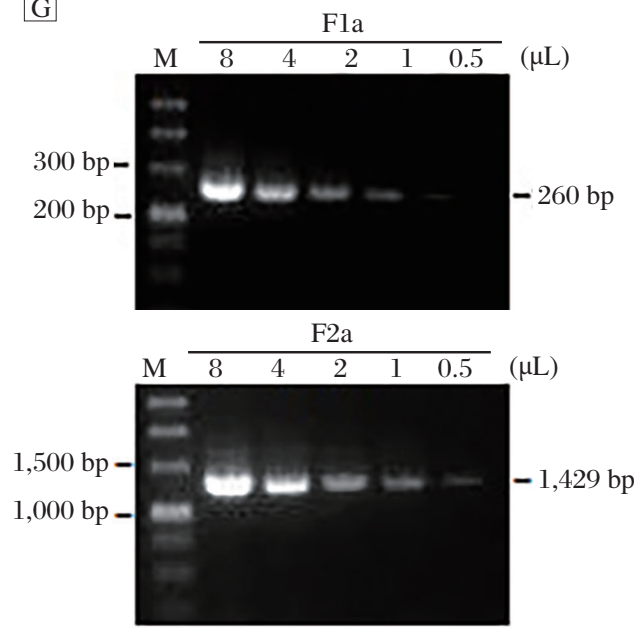

B

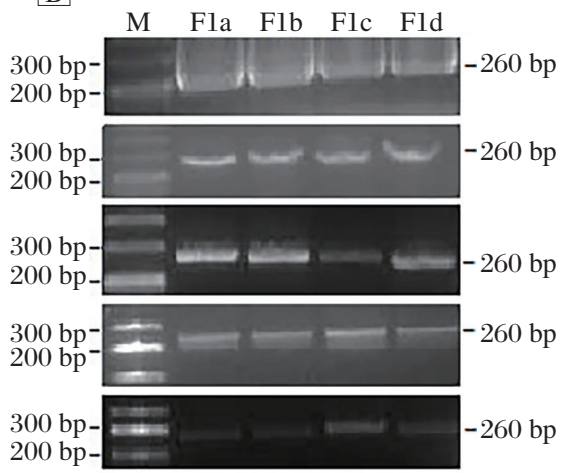

C

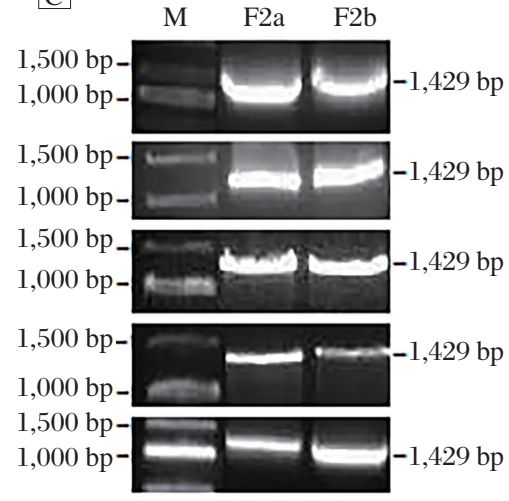

E

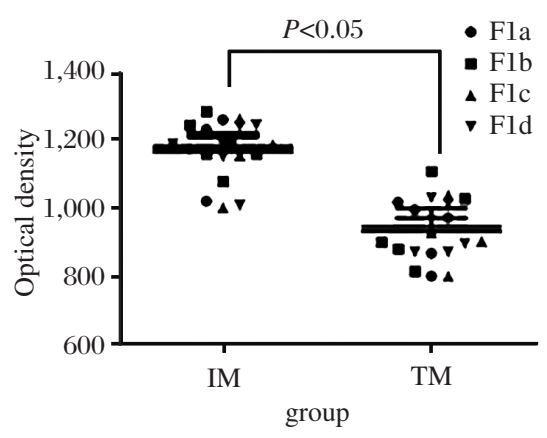

F

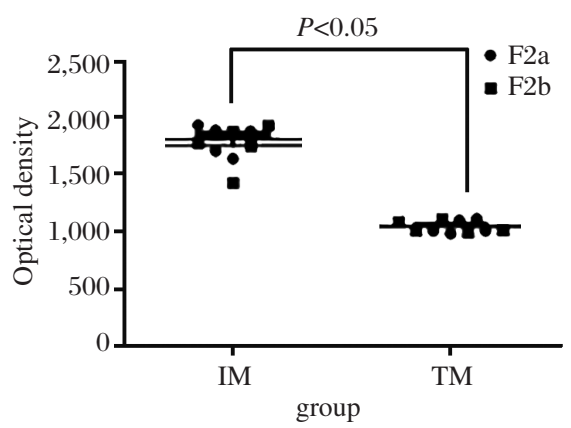

\begin{tabular}{lllllll}
$\mathrm{H}$ & \multicolumn{7}{c}{$\mathrm{Fla}$} \\
\cline { 3 - 6 } & $\mathrm{M}$ & 8 & 4 & 2 & 1 & 0.5
\end{tabular}
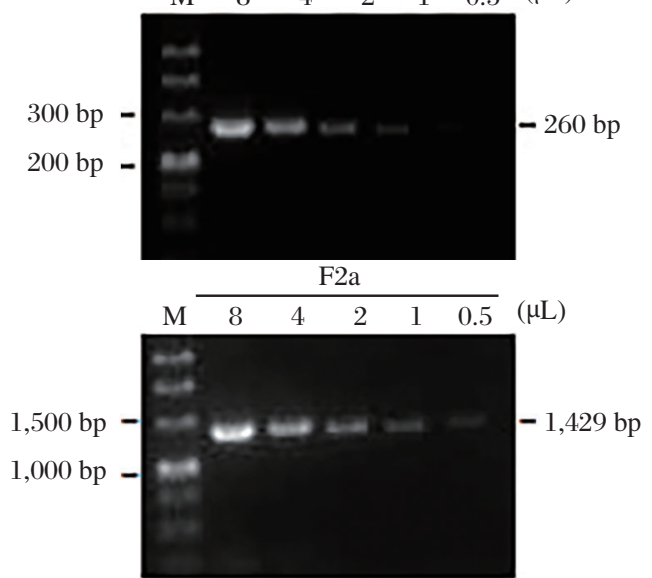

Fig. 1 The electrophoresis results of two different methods. A, B: the gel imaging results of fragment 1 by improved method (IM,A) and traditional method (TM,B); C, D: the gel imaging results of fragment 2 by improved method (IM,C) and traditional method (TM,D); E, F: the optical density of all bands by IM and TM $(P<0.05) ; \mathrm{G}, \mathrm{H}$ : the gel imaging results of two fragments by (IM,G) and $(\mathrm{TM}, \mathrm{H})$, both at different volumes $(\mu \mathrm{L})$ sign at top of pictures.Fla,Flb,Flc,Fld represent the fragment 1 from AGS,mKM,Sun719 and MKN45 cell; F2a,F2b represent the fragment 2 from AGS,Sun719 cells.

cleic acid sequences, 260 bp and 1,429 bp separately, representing short and long nucleic acid sequences, and the results were satisfied.

In conclusion, the improved method could reduce the amount of ethidium bromide used, minimize dye contact with human body, and obtain more obvious and reliable results. In the traditional method, there are towing bands behind the proof bands and unhelpful background resolution is higher. The improved meth- od has better results than the traditional method and can be used widely as a new technology in agarose gel electrophoresis.

\section{References}

[1] Lee PY, Costumbrado J, Hsu CY, et al. Agarose gel electrophoresis for the separation of DNA fragments. $J$ Vis Exp, 2012(62):e3923.

[2] Aaij C, Borst P. The gel electrophoresis of DNA. Biochim Biophys Acta, 1972,269(2):P192-200. 
[3] Huang Q, Fu W, Zhao Y, et al. Dyeing characteristics of nucleic acid fluorescent dyes in agarose gel electrophoresis. Chin J Nosocomiol(In Chinese), 2006(11):1316-8.

[4] Chang B, Zhang D, Li M, et al. Application of a novel nucleic acid dye to agarose gel electrophoresis. Modern Preventive Medicine(in Chinese), 2010(19):3717-20.

[5] Cong W, Chen M, Zhu Z, et al. A shortcut organic dyebased staining method for the detection of DNA both in agarose and polyacrylamide gel electrophoresis. Analyst, 2013,138(4):1187-94.

[6] Sanderson BA, Araki N, Lilley JL, et al. Modification of gel architecture and TBE/TAE buffer composition to minimize heating during agarose gel electrophoresis. Anal Biochem, 2014,454:44-52.

[7] Smith SB, Aldridge PK, Callis JB. Observation of individual DNA molecules undergoing gel electrophoresis. Science, 1989,243(4888):203-6.

[8] Haines AM, Tobe SS, Kobus HJ, et al. Properties of nucleic acid staining dyes used in gel electrophoresis. Electrophoresis, 2015, 36(6):941-4.

(Received 15 October 2016, Revised 08 November 2016, Accepted 10 December 2016) 